\title{
Far Lateral Tubular Decompression: A Case Series Studying One and Two Year Outcomes with Predictors of Failure
}

Ziyad O. Knio ${ }^{1}$, Wesley Hsu ${ }^{2}$, Alejandro Marquez-Lara ${ }^{1}$, Tianyi D. Luo ${ }^{3}$, John M. St.Angelo ${ }^{1}$ , Suman Medda ${ }^{1}$, Tadhg J. O'Gara ${ }^{1}$

1. Department of Orthopaedic Surgery, Wake Forest School of Medicine, Winston-Salem, USA 2. Department of Neurosurgery, Wake Forest School of Medicine, Winston-Salem, USA 3. Department of Orthopaedic Surgery, Wake Forest Baptist Medical Center, Winston-Salem, USA

$\square$ Corresponding author: Tadhg J. O'Gara, togara@wakehealth.edu Disclosures can be found in Additional Information at the end of the article

\section{Abstract}

\section{Introduction}

The optimal surgical treatment of isolated lumbar foraminal stenosis has not been defined. Minimally invasive decompression of the foramen from a far lateral tubular decompression (FLTD) approach has been shown to not only have minimal morbidity but also highly variable success rates at short-term follow-up. It is important to quantify improvement and define the demographic and radiographic parameters that predict failure in this promising, minimally invasive surgical technique. This study investigates pain and disability score improvement following FLTD at 12 and 24 months and investigates associations with failure.

\section{Methods}

All patients who underwent lumbar FLTD by a single surgeon at a single institution from September 2015 to January 2018 were included in this prospective case series. Visual analog scale (VAS) for back pain and leg pain and Oswestry Disability Index (ODI) were collected preoperatively and at the 12- and 24- month follow-ups. Outcomes between visits were fitted to a linear mixed-effects model. The univariate analysis investigated demographic, radiographic, and operative associations with subsequent open revision.

\section{Results}

A total of 42 patients were included in this study. Back pain (VAS 5.84 to 3.32, $\mathrm{p}<0.001$ ), leg pain (VAS 7.33 to 2.71, $\mathrm{p}<0.001$ ), and ODI (48.97 to 28.50, $\mathrm{p}<0.001$ ) demonstrated significant

Received 06/26/2019 Review began 06/29/2019 Review ended 07/03/2019 Published 07/13/2019

\section{(c) Copyright 2019}

Knio et al. This is an open access article distributed under the terms of the Creative Commons Attribution License CC-BY 3.0., which permits unrestricted use, distribution, and reproduction in any medium, provided the original author and source are credited. improvements at the 12-month follow-up. Back pain (VAS 3.71, p=0.004), leg pain (VAS 3.04, $\mathrm{p}<0.001)$, and ODI (30.63, $\mathrm{p}<0.001)$ improvements were maintained at 24-month follow-up. Four patients $(9.5 \%)$ required subsequent open revision. Subsequent open revision was associated with prior spine surgery $(\mathrm{RR}=2.85(2.07-3.63), \mathrm{p}=0.045)$ and scoliosis $\geqslant 10^{\circ}(\mathrm{RR}=6.33$ (4.87-7.80), $\mathrm{p}=0.013)$.

\section{Conclusion}

Back pain, leg pain, and ODI showed significant improvement postoperatively. Improvement is maintained at two years. Prior spine surgery and scoliosis $\geqslant 10^{\circ}$ may be relative contraindications to FLTD.

How to cite this article

Knio Z O, Hsu W, Marquez-Lara A, et al. (July 13, 2019) Far Lateral Tubular Decompression: A Case

Series Studying One and Two Year Outcomes with Predictors of Failure. Cureus 11(7): e5133. DOI $10.7759 /$ cureus.5133 
Categories: Neurosurgery, Orthopedics

Keywords: lumbar nerve decompression, tubular decompression, foraminal stenosis, minimally invasive surgery, disability, back pain, leg pain

\section{Introduction}

Foraminal stenosis causing radicular leg pain affects $8 \%-11 \%$ of lumbar spinal stenosis patients [1]. Unlike the more common causes of lumbar spinal stenosis in the central canal and lateral recesses, stenosis at the neuroforamen necessitates targeted surgical approaches due to its unique anatomy [2]. The optimal surgical approach for foraminal decompression continues to attract much debate. Symptomatic foraminal stenosis often exists concomitantly with the more common central and lateral recess varieties and, in these situations, the surgical approach must be tailored to address both anatomic locations. In situations where the foramen is the only location of pathology, a minimally invasive, outside-in approach can decompress the nerve root with minimal dissection and morbidity [3-6]. In the published literature, there are a few technical variants to this outside-in approach with a reported success rate ranging from $56 \%$ $88 \%$ in patients with radicular symptoms from neuroforaminal stenosis. Furthermore, factors associated with treatment failure include the L5-S1 level, higher coronal disc wedge angles, and higher segmental sagittal lordosis [4-5,7]. Despite great advances in minimally invasive surgical (MIS) techniques, there is limited data on the long-term outcomes associated with a far lateral MIS decompression and factors associated with symptomatic relief.

The purpose of this study was to investigate one- and two-year outcomes following far lateral tubular decompressions (FLTD) of the lumbar neuroforamen, as well as the patient and surgical factors associated with failure. We hypothesized that patients undergoing FLTD would demonstrate a significant improvement from presurgery to the 12-month follow-up and maintain the improvement at the 24-month follow-up.

\section{Materials And Methods}

This study was conducted at a single academic medical center with prospective data collection beginning in September 2015. Outcomes were collected at 12-month intervals as the standard of care. All aspects of the study were approved by the institutional review board. Consecutive patients with symptomatic degenerative lumbar foraminal stenosis who underwent FLTD by the senior author were included in this study.

\section{Patient selection}

Inclusion criteria for this study were patients $\geqslant 18$ years of age with symptomatic foraminal stenosis who underwent elective microdecompression of the lumbar spine. Patient selection for surgery was based on the preoperative evaluation by the senior author and the persistence of neurologic deficits after at least 90 days of conservative non-operative treatment (e.g., activity modification, non-steroidal anti-inflammatory drugs, physical therapy, and/or exercises). Selection criteria included the absence of a lytic spondylolisthesis at the affected level. Degenerative spondylolisthesis was not a contraindication to this approach. Magnetic resonance imaging (MRI) selection criteria included radicular symptoms that anatomically correlated with MRI evidence of nerve root compression in the foramen. The foramen size was not measured; instead, the nerve root was visualized on axial and sagittal T2 imaging throughout its course: If the root itself was compressed in the mid-zone or exit zone of the neuroforamen, the pathoanatomy was deemed amenable to far-lateral decompression. If significant lateral recess stenosis also involved this nerve root, patients were treated with a dual surgical approach: a tubular decompression laminotomy of the lateral recess combined with the far lateral approach. 


\section{Cureus}

\section{Surgical technique}

The surgical approach used was slightly different than the previously published techniques in that oblique fluoroscopy was used to approach the neuroforamen from a more lateral approach, sometimes up to $9 \mathrm{~cm}$ off midline $[5,8]$. This oblique approach permitted decompression of the foramen medial to the pedicle with minimal resection of the pars interarticularis (Figure 1). A surgical microscope was used in all cases. All patients were placed prone on a spine table and oblique fluoroscopy was used to dock the METRx ${ }^{\mathrm{TM}}$ tubular retractor (Medtronic, Dublin, Ireland) to the superior articular process (Figure 2). The foramen was then entered by burring the superolateral portion of the inferior vertebra's superior facet. Burring or resection of the pars interarticularis was not needed to gain access medial to the pedicle. With this technique, the skin incision ranged from $5-9 \mathrm{~cm}$ off midline.

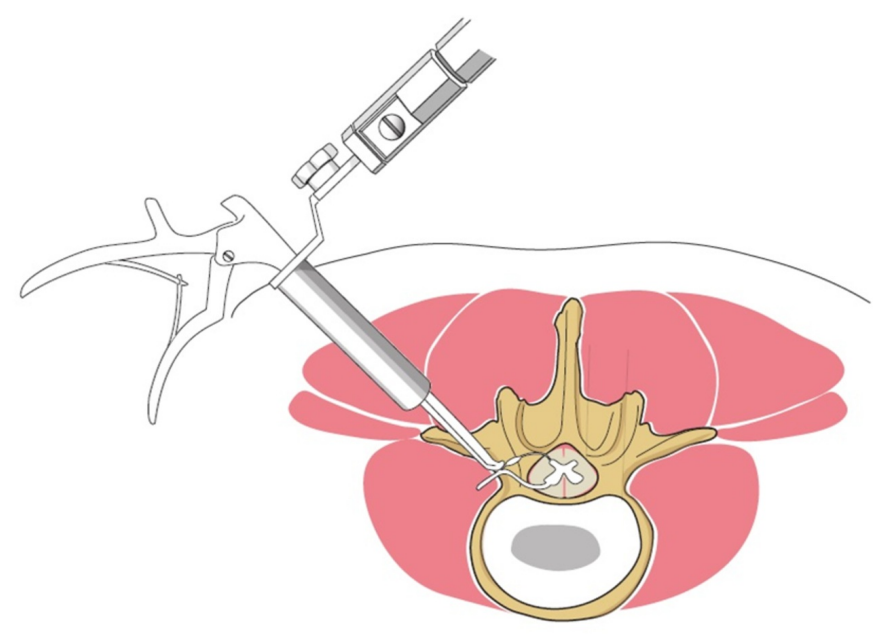

FIGURE 1: Far lateral tubular decompression approach.

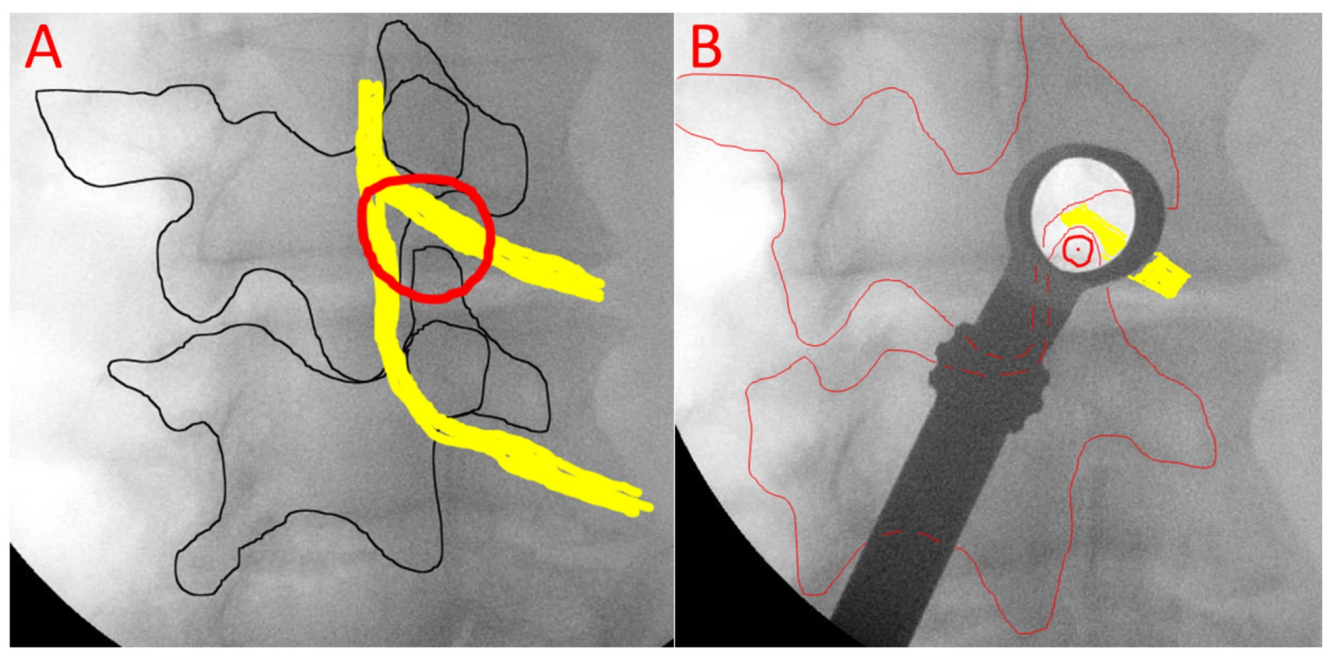

FIGURE 2: Intraoperative imaging with landmarks and 


\section{Data collection}

Visual analog scale (VAS) for back and leg pain and Oswestry Disability Index (ODI) were collected preoperatively and at 12-month intervals as the standard of care for all patients undergoing lumbar nerve decompression. Baseline scores were collected during preoperative office visits. Postoperative outcomes were either collected during follow-up office visits or via a standardized telephone script.

ODI is a composite score of the responses to 10 independent questions [9]. In cases where patients chose not to answer one or more questions, the score was computed from the available responses and scaled by a factor of $10 /$ ( 10 - \# of missing responses). No modifications were made to the raw score recorded for VAS.

Patient demographics (age, gender, BMI, current smoker, and diabetes diagnosis) and operative details were retrieved from the institutional electronic medical records. The operative time reported is the operative time per level; i.e. two-level decompression operative times were reduced by a factor of two. Spondylolisthesis, scoliosis, coronal wedge angle, and segmental lordosis angle measures from preoperative standing lumbar spine X-rays were assessed by a single reviewer. The coronal disc wedge angle of the operated segment was recorded as per the method used by Haimoto: "coronal angle between the line parallel to the lower endplate of the superior vertebra and the line parallel to the upper endplate of inferior vertebra" [4]. A positive angle referred to the operated side being closed down and on the concavity of the curve. The segmental lordosis angle at the operated level was recorded. At L5-S1, this corresponded to the angle created by lines through the superior endplates of L5 and S1. At the other lumbar levels, this corresponded to the angle created by lines through the superior endplate of the superior vertebra and the inferior endplate of the inferior vertebra.

\section{Statistical methods}

All computations and statistical analyses were performed in R (R Core Team, Vienna, Austria). A linear mixed-effects model was fitted to outcomes and preoperative scores, controlling for differences between patients. Pairwise differences were investigated with a post-hoc Tukey test for multiple comparisons. This methodology allowed for an investigation of whether pain and disability scores demonstrated improvement from pre-surgery to 12-month follow-up, whether the improvement was maintained at 24-month follow-up, and whether there was any deterioration between 12-month and 24-month follow-up. A p-value of 0.05 was considered significant for outcomes improvement.

Patient demographic, radiographic, and operative factors were compared between those who did and did not require subsequent open revision. These comparisons were quantified with a Mann-Whitney U test for continuous variables and with Pearson's Chi-Square Test for categorical variables.

\section{Results}

A total of 42 consecutive patients who underwent FLTD were included in the study. Given the prospective nature of the data collection, 28 patients were eligible for two-year outcomes inclusion and 14 patients were eligible for only one-year outcomes inclusion. Four patients (9.5\%) underwent multi-level FLTD. Eight patients (19.0\%) had simultaneous discectomy. Two patients (4.8\%) required a dual surgical approach (tubular decompression laminotomy of the lateral recess combined with the far-lateral approach) to address lateral recess stenosis. A 


\section{Cureus}

summary of patient characteristics is presented in Table 1.

\begin{tabular}{|c|c|c|}
\hline \multirow{3}{*}{ Variable } & Mean \pm SD & \multirow{3}{*}{$\mathbf{n}$} \\
\hline & $p(\%)$ & \\
\hline & $n=42$ & \\
\hline Age (years) & $67.50 \pm 12.90$ & 42 \\
\hline Wredge angle (") & $2.15 \pm 2.39$ & 34 \\
\hline Segmental lordosis $\left(^{\circ}\right)$ & $21.41 \pm 7.82$ & 34 \\
\hline Operative time (minutes) & $90.09 \pm 34.28$ & 41 \\
\hline Length of stay (days) & $0.76 \pm 1.15$ & 42 \\
\hline Gender (male) & 25/42 (59.5\%) & 42 \\
\hline BMI>30 & $19 / 40(47.5 \%)$ & 40 \\
\hline Current smoker & 4/42 (9.5\%) & 42 \\
\hline Diabetes & $8 / 42(19.0 \%)$ & 42 \\
\hline Synovial cyst & $3 / 42(7.1 \%)$ & 42 \\
\hline Multiple levels & $4 / 42(9.5 \%)$ & 42 \\
\hline Prior spine surgery & 13/42 (31.0\%) & 42 \\
\hline Disc procedure & $8 / 42(19.0 \%)$ & 42 \\
\hline Laminotomy & $2 / 42(4.8 \%)$ & 42 \\
\hline Spondylolisthesis $\geq 4 \mathrm{~mm}$ & 6/42 (14.3\%) & 42 \\
\hline Scoliosis $\geq 10^{\circ}$ & $5 / 42(11.9 \%)$ & 42 \\
\hline
\end{tabular}

\section{TABLE 1: Patient characteristics of FLTD cases.}

BMI: body mass index, FLTD: far lateral tubular decompression, p: proportion, SD: standard deviation

Back pain (VAS 5.84 to 3.32, $\mathrm{p}<0.001$ ), leg pain (VAS 7.33 to $2.71, \mathrm{p}<0.001$ ), and ODI (48.97 to $28.50, \mathrm{p}<0.001)$ demonstrated statistically significant improvements at the 12-month followup. Back pain (VAS 3.71, p=0.004), leg pain (VAS 3.04, p<0.001), and ODI (30.63, p<0.001) improvements were maintained at 24 months. No outcomes deteriorated between the 12month and the 24-month follow-up ( $>0.050$; Table 2, Figure 3). 


\section{Cureus}

\begin{tabular}{|c|c|c|c|c|c|c|c|c|c|}
\hline \multirow{2}{*}{ Variable } & \multicolumn{2}{|l|}{ Pre-surgery } & \multicolumn{2}{|c|}{12 month follow-up } & \multicolumn{2}{|c|}{24 month follow-up } & \multirow{2}{*}{$\begin{array}{l}\text { pre - } 12 \\
\text { p-value }\end{array}$} & \multirow{2}{*}{$\begin{array}{l}\text { pre - } 24 \\
\text { p-value }\end{array}$} & \multirow{2}{*}{$\begin{array}{l}12-24 \\
p \text {-value }\end{array}$} \\
\hline & Mean \pm SD & $\mathrm{n}$ & Mean \pm SD & $\mathrm{n}$ & Mean \pm SD & $\mathrm{n}$ & & & \\
\hline Back Pain & $5.84 \pm 2.94$ & 38 & $3.32 \pm 2.81$ & 38 & $3.71 \pm 2.93$ & 24 & $<0.001^{*}$ & $0.004^{*}$ & 0.694 \\
\hline Leg Pain & $7.33 \pm 2.29$ & 39 & $2.71 \pm 2.99$ & 38 & $3.04 \pm 2.76$ & 24 & $<0.001^{*}$ & $<0.001^{*}$ & 0.796 \\
\hline ODI & $48.97 \pm 20.92$ & 37 & $28.50 \pm 19.71$ & 38 & $30.63 \pm 18.45$ & 24 & $<0.001^{*}$ & $<0.001^{*}$ & 0.732 \\
\hline
\end{tabular}

\section{TABLE 2: Comparisons of pre-surgery, 12 month, and 24 month follow-up outcomes for FLTD cases.}

*: p<0.05, FLTD: far lateral tubular decompression, ODI: Oswestry Disability Index, SD: standard deviation

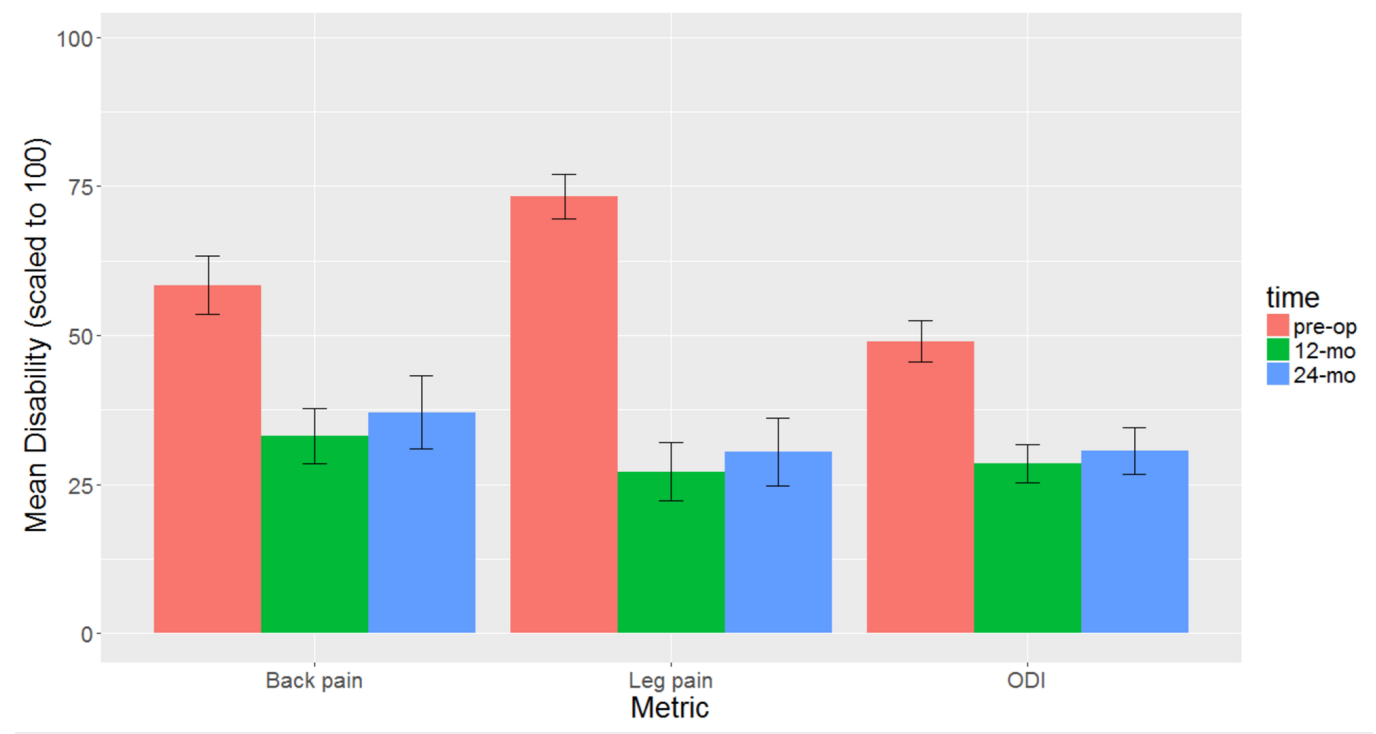

FIGURE 3: Back pain, leg pain, and Oswestry Disability Index vs. time.

Four patients (9.5\%) required subsequent open revision decompression and fusion. These four patients underwent isolated FLTD during the first year of the study investigation, with dates of surgery ranging from December 2015 to May 2016. No patients since then have required subsequent open revision. The subsequent open revisions were performed at a median of 9.5 months after index microdecompression (range two months - 24 months). Three of the four patients had complex spine pathologies (i.e. occult pars fracture) and a history of prior spine surgery (i.e. emergency decompression for cauda equina syndrome, four-level cervical fusion). The fourth patient had $15^{\circ}$ of lumbar spine scoliosis and required a multi-level decompression.

Six patients (17.6\%) required a subsequent minimally invasive procedure after isolated FLTD. These cases were not considered failures, as patients acknowledged and accepted that this may be warranted. Moreover, these patients generally experienced initial relief, with symptoms recurring months or even years later. Subsequent microdecompression was performed at a 


\section{Cureus}

median of 17 months after index microdecompression (range seven months - 24 months). Three of the six patients exhibited pathology recurring at the same level and side but inside the canal at the lateral recess rather than outside the foramen. One patient was an elderly patient with multiple prior surgeries who did not improve clinically. The remaining two patients developed pathological problems at a level different than that of their initial presentation.

Subsequent open revision was associated with prior spine surgery ( $R R=2.85$ (2.07-3.63), $\mathrm{p}=0.045)$ and scoliosis $\geqslant 10^{\circ}(\mathrm{RR}=6.33$ (4.87-7.80), $\mathrm{p}=0.013$; Table 3).

\begin{tabular}{|c|c|c|c|c|c|}
\hline \multirow{4}{*}{ Variable } & No need for major revision & Subsequent major revision & \multirow{4}{*}{$\mathbf{n}$} & \multirow{4}{*}{\multicolumn{2}{|c|}{ p-value }} \\
\hline & Mean \pm SD & Mean \pm SD & & & \\
\hline & $p(\%)$ & $\mathrm{p}(\%)$ & & & \\
\hline & $\mathrm{n}=38$ & $\mathrm{n}=4$ & & & \\
\hline Age (years) & $67.89 \pm 13.38$ & $63.75 \pm 6.70$ & 42 & 0.493 & \\
\hline Wedge angle $\left({ }^{\circ}\right)$ & $2.10 \pm 2.39$ & $2.67 \pm 2.89$ & 34 & 0.636 & \\
\hline Segmental lordosis $\left({ }^{\circ}\right)$ & $21.94 \pm 7.80$ & $16.00 \pm 7.00$ & 34 & 0.224 & \\
\hline Operative time (minutes) & $90.88 \pm 35.43$ & $82.75 \pm 22.97$ & 41 & 0.982 & \\
\hline Length of stay (days) & $0.78 \pm 1.20$ & $0.56 \pm 0.47$ & 42 & 0.813 & \\
\hline Gender (male) & $23 / 38(60.5 \%)$ & $2 / 4(50.0 \%)$ & 42 & 0.683 & \\
\hline $\mathrm{BMI}>30$ & $18 / 36(50.0 \%)$ & $1 / 4(25.0 \%)$ & 40 & 0.342 & \\
\hline Current smoker & $3 / 38(7.9 \%)$ & $1 / 4(25.0 \%)$ & 42 & 0.268 & \\
\hline Diabetes & $8 / 38(21.1 \%)$ & $0 / 4(0 \%)$ & 42 & 0.308 & \\
\hline Synovial cyst & $3 / 38(7.9 \%)$ & $0 / 4(0 \%)$ & 42 & 0.560 & \\
\hline Multiple levels & $3 / 38(7.9 \%)$ & $1 / 4(25.0 \%)$ & 42 & 0.268 & \\
\hline Prior spine surgery & 10/38 (26.3\%) & $3 / 4(75.0 \%)$ & 42 & 0.045 & * \\
\hline Disc procedure & $7 / 38(18.4 \%)$ & $1 / 4(25.0 \%)$ & 42 & 0.750 & \\
\hline Laminotomy & $2 / 38(5.3 \%)$ & $0 / 4(0 \%)$ & 42 & 0.638 & \\
\hline Spondylolisthesis $\geq 4 \mathrm{~mm}$ & 6/38 (15.8\%) & $0 / 4(0 \%)$ & 42 & 0.391 & \\
\hline Scoliosis $\geq 10^{\circ}$ & $3 / 38$ (7.9\%) & $2 / 4(50.0 \%)$ & 42 & 0.013 & * \\
\hline
\end{tabular}

\section{TABLE 3: One-way associations with need for revision of index FLTD cases.}

*: p<0.05, BMI: body mass index, FLTD: far lateral tubular decompression, p: proportion, SD: standard deviation

\section{Discussion}


The present study highlights both the benefits and challenges of utilizing FLTD, a minimally invasive, same-day surgery to treat symptomatic degenerative lumbar foraminal stenosis. Significant reductions in back pain, leg pain, and ODI were demonstrated postoperatively and maintained at two years. The current literature defining minimum clinically important differences $(1.2,1.6$, and 12.8 for back pain, leg pain, and ODI, respectively) suggests that the improvement observed in this study is not only statistically significant but also clinically significant [10]. Patients who failed the treatment were successfully treated with facet resection and fusion. Prior spine surgery and scoliosis $\geqslant 10^{\circ}$ were significantly associated with surgical failure requiring a secondary procedure.

Numerous studies have described paramedian or far-lateral decompression techniques with varying results [4-8]. Kim et al. evaluated extraforaminal decompression without fusion using a paramedian incision coming directly down on the pars interarticularis and compared this technique to posterior lumbar interbody fusion (PLIF) [8]. They reported a revision rate of $12 \%$ (3/25 patients) in the decompression group and concluded that the extraforaminal decompression yielded results at least as good as PLIF. Yamada et al. also used a paramedian approach with partial pars resection at the foramen and reported $20 \%$ treatment failure at follow-up [5]. Of the failures, $89 \%$ had degenerative lumbar scoliosis, which the authors deemed a significant risk factor for treatment failure. In our study, we were able to quantify scoliosis $\geqslant 10^{\circ}$ as a significant risk factor for surgical failure. Hari et al. described a minimally invasive lateral foraminotomy using tubular retractors with partial lateral facetectomy [11]. None of 12 patients investigated required additional surgery at a follow-up period of at least one year. Chang et al. described a microsurgical foraminotomy via a posterolateral transmuscular approach and a contralateral oblique approach, with excellent or good results by the MacNab Scale in 33 of 39 patients (85\%), at a mean follow-up of 25.5 years [12]. Haimoto et al. evaluated 12 far-lateral decompressions at an average follow-up of 19 months and showed a revision rate of $42 \%$ (5/12 patients) [4]. Risk factors for failure included an increased preoperative coronal plane disc wedge angle of the L5-S1 segment (3.5 degrees versus 1.1 degrees in the success group). Cho et al. described an open far-lateral, muscle-splitting approach to L5-S1 in 21 patients [7]. At an average of 18 months' follow-up, 33\% failed to demonstrate clinical improvement. Treatment failure was associated with higher segmental lordosis angles in a neutral upright posture $\left(18.4^{\circ}\right.$ vs. $\left.13^{\circ}, \mathrm{p}=0.02\right)$. Unlike Haimoto and Cho, we did not see an association between coronal wedge and segmental lordosis angles.

From a clinical standpoint, foraminal stenosis remains a challenging problem to address, particularly utilizing minimally invasive techniques. Continued movement of the segment with disc bulging and facet hypertrophy may well contribute to the recurrence of symptoms. In a registry study of MIS transforaminal decompressions by Sclafani et al., the authors reported a revision rate of $2 \%$ for those undergoing discectomy and $28 \%$ for those undergoing decompression from bony foraminal stenosis [6]. Furthermore, patients who underwent a discectomy demonstrated a significant improvement in ODI (19.4 points, $\mathrm{p}=0.0002)$ while those with bony foraminal stenosis only improved by 7.1 points $(p=0.06)$. Bony foraminal stenosis from low-grade spondylolisthesis requires adequate intraoperative decompression without compromising facet joint stability. These technical challenges may have contributed to the differences seen in clinical outcomes in the study by Sclafani et al. [6]. In the present study, the need for simultaneous discectomy (8/42) or the presence of a low-grade spondylolisthesis (6/42) was not significantly associated with adverse outcomes.

The statistical significance of the VAS and ODI score improvements is likely reflective of a clinical significance, with the ODI minimum clinically important difference estimated to range between 2.92 to 15.36, depending on the calculation method [10]. Chung et al. described the current trends of defining clinical improvements in spine surgery and highlighted the need for future studies to better define the "clinical importance" of a change in patient-reported outcomes [13]. Taking into account the current improvement criteria, this approach appears to 
provide patients the option to significantly alleviate pain and regain functionality with a minimally invasive, same-day surgery. As the trend to value-based healthcare system continues to evolve, discussing the limitations of this technique, as well as addressing realistic expectations, is important to optimize patient satisfaction.

This case series is limited by the small sample size. Large database studies aside, this is characteristic of studies investigating minimally invasive decompressions. A control group was not included for analysis. Ideally, a non-surgical group would serve as a control, however, this has the potential to compromise patient care and may introduce selection bias to the study. As such, this study aimed to describe outcomes at one-year and two-year follow-up rather than to demonstrate superiority to non-operative management or other surgical techniques. Furthermore, all procedures were performed by a single surgeon; however, we believe the technique is reproducible, and that this limitation does not affect the generalizability of the results. A strength of this study is the prospective nature of the data collection, which allows for the identification of predictors of failure using a novel technique.

\section{Conclusions}

In patients with symptomatic degenerative lumbar foraminal stenosis who underwent FLTD of the lumbar neuroforamen, back pain, leg pain, and disability scores showed consistent improvement at two years. Based on our reported outcomes and complications, prior spine surgery and scoliosis $\geqslant 10^{\circ}$ may be considered relative contraindications to this technique. Continued outcomes collection at five years postoperatively will better describe the long-term outcomes of this approach.

\section{Additional Information}

\section{Disclosures}

Human subjects: Consent was obtained by all participants in this study. Wake Forest University Health Sciences Institutional Review Board issued approval IRB00042783. This is to confirm for your record that the Institutional Review Board reviewed your progress report and consent form, containing compounded HIPAA authorization language, if applicable, for the above-named protocol. IRB approval was activated on 12/24/2018 and will expire on $12 / 23 / 2019$. If the protocol is to remain active longer, a written request for renewal, together with a summary progress report, and a copy of the current consent form, if applicable, should be submitted to the Board at least one month prior to expiration. Upon review of the research, the IRB finds that this study is classified as Expedited Category 5. Upon review of the research, the IRB finds that this study is classified as Expedited Category 7. This research meets criteria for a waiver of written (signed) consent according to 45 CFR 46.117(c)(2). This research meets the criteria for a waiver of HIPAA authorization according to 45 CFR 164.512. Please provide a final report to the Board when the project is completed and Board approval can be terminated. This IRB is in compliance with the requirements in Part 56, Subchapter D, Part 312 of the 21 Code of Federal Regulations published January 27, 1981 and Part 46, Subpart A of 45 CFR published January 26, 1981. . Animal subjects: All authors have confirmed that this study did not involve animal subjects or tissue. Conflicts of interest: In compliance with the ICMJE uniform disclosure form, all authors declare the following: Payment/services info: All authors have declared that no financial support was received from any organization for the submitted work. Financial relationships: All authors have declared that they have no financial relationships at present or within the previous three years with any organizations that might have an interest in the submitted work. Other relationships: All authors have declared that there are no other relationships or activities that could appear to have influenced the submitted work. 


\section{Acknowledgements}

We thank Wendy Williams and Marcy Lewis for their assistance in outcomes collection.

\section{References}

1. Rothman RH, Simeone FA: Rothman-Simeone the Spine (5th ed.). Herkowitz HN, Garfin SR, Eismont FJ, Bell GR, Balderson RA (ed): Saunders-Elsevier, Philadelphia; 2006.

2. Epstein NE: Case presentation and short perspective on management of foraminal/far lateral discs and stenosis. Surg Neurol Int. 2018, 9:87. 10.4103/sni.sni_66_18

3. Alimi M, Njoku I Jr, Cong GT, Pyo SY, Hofstetter CP, Grunert P, Hartl R: Minimally invasive foraminotomy through tubular retractors via a contralateral approach in patients with unilateral radiculopathy. Neurosurgery. 2014, 3:446-437. 10.1227/NEU.0000000000000358

4. Haimoto S, Nishimura Y, Hara M, Nakajima Y, Yamamoto Y, Ginsberg HJ, Wakabayashi T: Clinical and radiological outcomes of microscopic lumbar foraminal decompression: a pilot analysis of possible risk factors for restenosis. Neurol Med Chir (Tokyo). 2018, 58:49-58. 10.2176/nmc.oa.2017-0121

5. Yamada K, Matsuda H, Nabeta M, Habunaga H, Suzuki A, Nakamura H: Clinical outcomes of microscopic decompression for degenerative lumbar foraminal stenosis: a comparison between patients with and without degenerative lumbar scoliosis. Eur Spine J. 2011, 20:947953. 10.1007/s00586-010-1597-1

6. Sclafani JA, Raiszadeh K, Laich D, et al.: Outcome measures of an intracanal, endoscopic transforaminal decompression technique: initial findings from the MIS prospective registry. Int J Spine Surg. 2015, 9:69. 10.14444/2069

7. Cho SI, Chough CK, Choi SC, Chun JY: Microsurgical foraminotomy via Wiltse paraspinal approach for foraminal or extraforaminal stenosis at L5-S1 level: risk factor analysis for poor outcome. J Korean Neurosurg Soc. 2016, 59:610-614. 10.3340/jkns.2016.59.6.610

8. Kim HJ, Jeong JH, Cho HG, Chang BS, Lee CK, Yeom JS: Comparative observational study of surgical outcomes of lumbar foraminal stenosis using minimally invasive microsurgical extraforaminal decompression alone versus posterior lumbar interbody fusion: a prospective cohort study. Eur Spine J. 2014, 24:388-395. 10.1007/s00586-014-3592-4

9. Fairbank JC, Pynsent PB: The Oswestry Disability Index. Spine. 2000, 25:2940-2952.

10. Copay AG, Glassman SD, Subach BR, Berven S, Schuler TC, Carreon LY: Minimum clinically important difference in lumbar spine surgery patients: a choice of methods using the Oswestry Disability Index, Medical Outcomes Study questionnaire Short Form 36, and pain scales. Spine J. 2008, 8:968-974. 10.1016/j.spinee.2007.11.006

11. Hari A, Krishna M, Rajagandhi S, Sharma A, Deshpande RV: Minimally invasive lateral foraminotomy with partial lateral facetectomy for lumbar radiculopathy- An evaluation of facet integrity and description of the procedure. Neurol India. 2017, 65:1358-1365. 10.4103/0028-3886.217932

12. Chang HS, Zidan I, Fujisawa N, Matsui T: Microsurgical posterolateral transmuscular approach for lumbar foraminal stenosis. J Spinal Disord Tech. 2011, 24:302-307. 10.1097/BSD.0b013e3181f7cc9f

13. Chung AS, Copay AG, Olmscheid N, Campbell D, Walker JB, Chutkan N: Minimum clinically important difference: current trends in the spine literature. Spine. 2017, 42:1096-1105. 10.1097/BRS.0000000000001990 